\title{
Un modelo predictivo del comportamiento de la práctica deportiva entre la generación Millennial A predictive model of sports practice behavior among the Millennial generation
}

\author{
Alazne Mujika-Alberdi, Juan José Gibaja-Martins, Iñaki García-Arrizabalaga \\ Universidad de Deusto (España)
}

\begin{abstract}
Resumen. Esta investigación tiene por objetivo diseñar un modelo de predicción del comportamiento de la práctica deportiva a partir de la escala de actitud hacia la actividad física y el deporte previo contraste psicométrico entre la generación Millennial. Además, profundiza en la medición de la actitud hacia la actividad física y el deporte y en la determinación de diferencias en función de género y nivel de práctica deportiva. El trabajo de campo se realizó sobre una muestra de 1141 individuos de la generación Millennial seleccionados por muestreo de cuotas de género y edad. El estudio avanza respecto a la literatura existente apuntando una bi-dimensionalidad de la escala y aporta un modelo predictivo para la población Millennial con una elevada tasa de éxito para clasificar individuos practicantes y no practicantes a partir de la aplicación de la técnica de regresión logística. Esta mirada a los Millennial tiene su valor no tanto en la comparación con otras generaciones sino en la necesidad de predecir comportamientos futuros, siendo esencial desarrollar actitudes que favorezcan hábitos saludables beneficiosos para el individuo y la comunidad en su conjunto. Adicionalmente, los resultados muestran cierta novedad al descubrir particularidades de los Millennials donde la actitud hacia el ejercicio físico y la práctica deportiva es mejor entre las mujeres que entre los hombres.
\end{abstract}

Palabras Clave. Modelo predictivo, actitud, comportamiento, actividad física, práctica deportiva, Millennials.

Summary. This research aims to design a model for predicting the behavior of sports practice for the Millennial generation. After psychometric contrast, the study is based on the scale of attitude towards physical activity and sport. In addition, the research deepens in the measurement of the attitude towards physical activity and sport, and explores the existence of differences regarding gender and level of practice. Anon-random sample of 1141 Millennial generation individuals selected with gender and age quotas was surveyed. The study goes forward with respect to the existing literature, suggesting a bi-dimensionality of the used scale. It also provides a predictive model for Millennial generation, based on the application of the logistic regression technique, with a high success rate to classify sport practicing and non-practicing individual. This glance at Millennials lays its value in the need of predicting future behaviors rather than comparing with other generations. In this sense, the development of attitudes that promote healthy habits, which are also beneficial both to individuals and the community as a whole is essential. Additionally, results show some novelty concerning particularities of Millennials: their attitude towards physical exercise and sports practice is better among women than men.

Key words. Predictive model, attitude, physical activity, behavior, sports practice, Millennials.

\section{Introducción}

La generación Millennial, también conocida como generación $\mathrm{Y}$, es la cohorte demográfica que sigue a la generación $\mathrm{X}$ y que, a pesar del disenso a la hora de establecer las fechas de inicio y fin de la misma, se dice de aquellos nacidos entre el año 1980 y 2000. Según Eurostat, en 2017 más de 100 millones de ciudadanos de la Unión Europea eran Millennials, esto es, el 20\% de la población. En España son el 19\% de la población.

Se dice de ellos que son la generación más preparada. Según Eurostat, en 2017 cerca del 40\% de adultos jóvenes de Europa tenían estudios de grado superior, la cifra más alta de todos los tiempos. Los Millennials también son descritos como «nativos digitales», «multipantalla y multidispositivo», «nomófobos y appdictos», extremadamente sociales y «super conectados», críticos, exigentes y volátiles (BBVA Innovation Center, 2015). El estudio de la Fundación Mapfre (2019) pone también de manifiesto su preocupación por la salud tanto física como mental y su interés por mantener unos hábitos saludables. En esta línea, según el estudio de Nielsen Sports (2018), los Millennials muestran un alto interés por el deporte, detrás de ir al cine y ver películas y series o asistir a eventos musicales, y por delante de ver la televisión, moda, jugar a videojuegos, salir de copas, el arte o

Fecha recepción: 14-02-20. Fecha de aceptación: 28-05-20

Alazne Mujika Alberdi

alazne.mujika@deusto.es acudir al teatro, ópera o ballet. Además, según este mismo estudio, el $72 \%$ dice practicar deporte con una frecuencia de al menos dos o tres días por semana, siendo los deportes más practicados ir al gimnasio y correr, en este orden. En este mismo sentido, Fraguela-Vale, Varela-Garrote \& Varela-Crespo (2020) contrastaron que, en general, la juventud española prioriza el deporte para disfrutar de su tiempo de ocio. De todas formas, el riesgo de un estilo de vida poco activo de los jóvenes «multipantalla» y «super conectados» es la reducción de la actividad física por la relación existente entre estilo de vida y el comportamiento (Meneses \& Ruiz, 2017, Gómez, Sánchez \& Labisa, 2020). Sin embargo, también se sabe que la actividad física decrece al iniciar la edad adulta. Así lo recoge, por ejemplo, la Encuesta de Hábitos Deportivos en España (Ministerio de Educación, Cultura y Deporte, 2015).

La actividad física y la práctica deportiva regular son hábitos que protegen contra muchas enfermedades, previenen el aumento de peso, sobrepeso y obesidad, y coadyuvan a mantener un estilo de vida saludable. Según el Fondo Mundial para la Investigación del Cáncer/Instituto Estadounidense de Investigación sobre el Cáncer, en su informe Alimentos, nutrición, actividad física, y la prevención del cáncer: una perspectiva mundial, a medida que los países adopten un estilo de vida «occidental», la incidencia del cáncer podría incrementarse en 24 millones de nuevos casos en todo el mundo para 2035. Para prevenirlo sugiere tener una dieta saludable, eliminar el tabaco, mantener un peso saludable y hacer ejercicio regular, pues las pruebas científicas re- 
cogidas en dicho informe demuestran la existencia de nexos entre muchos tipos de enfermedad y la vida sedentaria. El fomento del ejercicio físico y la práctica deportiva es, pues, prioritario para el bienestar individual y comunitario.

Una de las cuestiones que influye positivamente en la práctica deportiva es, justamente, la actitud hacia la actividad física y deportiva (véanse, por ejemplo, Tereza Araújo \& Dosil, 2016, y los trabajos citados por ellos como Lima, 2000; Araújo, Calmeiro \& Palmeira, 2005; Caetano \& Raposo, 2005; Martin, Oliver \& McCaughtry, 2007; Biddle y Mutrie, 2008; o Fermino, Pezzini \& Reis, 2010). Además, se ha contrastado que la actitud es un buen predictor del comportamiento (Hagger, Chatzisanrantis \& Biddle, 2001).

Por todo ello surge el interés por profundizar en la actitud hacia la actividad física y deportiva de esta particular generación, la de los Millennials. La escala de Dosil (2002) ha demostrado ser un instrumento fiable y válido de la actitud hacia la actividad física y deportiva que, además, está contrastado en diferentes ámbitos geográficos y distintas poblaciones (véanse, por ejemplo, Tereza Araújo \& Dosil, 2015; Tereza Araújo \& Dosil, 2016). Esta escala pretende cubrir una carencia de otras al incorporar ítems orientados a los individuos practicantes y a los no-practicantes, pues el autor de la escala, Dosil, entendía que la actitud hacia la actividad física y el deporte podría ser favorable tanto en un caso como en otro. En la literatura se anima, también, a estudiar la existencia de diferencias en la actitud entre los practicantes y nopracticantes siguiendo la estela de autores como Ruiz, García \& Diaz (2007), Haase \& Kinnafick (2007) o Sicilia, Águila, Muyor, Orta \& Moreno (2009). Asimismo, el autor de la escala propone contrastar la existencia de diferencias en la actitud entre hombres y mujeres, atendiendo a los resultados de otros muchos autores como Salles-Costa (2003), Pavón \& Moreno (2008), Cid \& Alves (2008), Cid, Chicau, Silva \& Moutao (2009), Ornelas-Melim \& Oliveira-Pereira (2013) o Herazo-Beltran, Nuñez-Bravo, Sánchez-Guette, VásquezOsorio, Lozano-Ariza, Torres-Herrera \& Valdelamar-Villegas (2020).

Por tanto, el propósito de este trabajo es doble, metodológico y de medición. A nivel metodológico, se trata de, en primer lugar, contrastar la fiabilidad y validez de la escala de Dosil (2002) en la población Millennial y, en segundo lugar, proponer un modelo capaz de predecir el comportamiento futuro de la práctica deportiva en base a la actitud hacia la actividad física y el deporte. A nivel de medición, se quiere conocer la actitud hacia la actividad física y deportiva de los Millennials, determinar la existencia de diferencias de esta actitud entre los practicantes y no-practicantes de actividad física y deportiva e identificar la existencia de diferencias entre hombres y mujeres de este grupo de población.

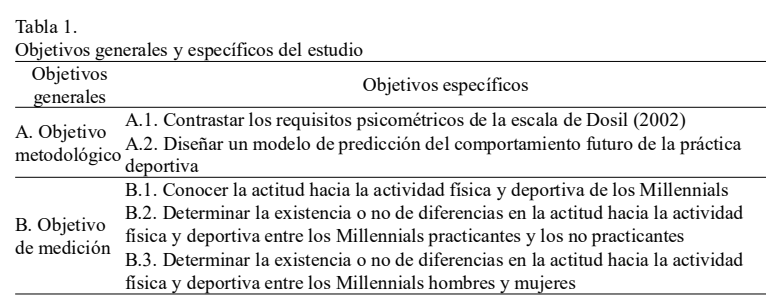

Tener un modelo predictivo del comportamiento deportivo a partir de una escala fiable y válida de la actitud y cono- cer la existencia de diferencias entre grupos ayudará a determinar estrategias, políticas, programas y acciones que promocionen la práctica regular de actividad física y deportiva y, consecuentemente, promoverá la salud individual y comunitaria. No cabe duda de que conocer gustos, intereses, comportamientos, hábitos de consumo, usos de tiempo de ocio, incluidos los del entretenimiento, cultura y deporte, $\mathrm{y}$, en definitiva, la manera de interpretar la realidad de los Millennials es un reto para las diferentes estructuras económicas, sociales, culturales, políticas y mass media. Profundizar en estos objetivos ayudará a las entidades públicas y privadas a prever escenarios futuros y tomar las mejores decisiones posibles.

\section{Material y método}

Para dar respuesta a los objetivos enunciados previamente se diseñó un cuestionario. La revisión de estudios similares publicados en revistas científicas especializadas como la Revista de Psicología del Deporte, Revista Internacional de Ciencias del Deporte, Revista Internacional de Medicina y Ciencias de la Actividad Física y el Deporte, Apunts, Retos o la Encuesta de Hábitos Deportivos en España publicada por el Ministerio de Educación, Cultura y Deporte, llevó a los investigadores a decantarse por la utilización de la escala de Dosil (2002), ya validada en otras poblaciones, y que mide la actitud hacia la actividad física y práctica deportiva. Este instrumento consta de doce ítems medidos en una escala Likert de cinco puntos, desde totalmente en desacuerdo (puntuación uno) hasta totalmente de acuerdo (puntuación cinco).

El borrador de cuestionario se sometió a una prueba piloto compuesta por una docena de Millennials. Esto permitió corregir errores de comprensión en su enunciado y se pudo comprobar que funcionaba correctamente.

La población objeto de estudio de esta investigación fue la formada por los guipuzcoanos nacidos entre el año 1980 y 2000, esto es, los denominados Millennials. Según datos de Eustat (2017), el número de guipuzcoanos Millennials asciende a cerca de 150000 , de los que el $49,1 \%$ son mujeres, y suponen cerca del $20 \%$ de la población guipuzcoana total. La tabla 2 muestra el detalle. A partir de los datos del Instituto Vasco de Estadística, EUSTAT (www.eustat.eus), se obtuvo el perfil de la población de Gipuzkoa según las variables de sexo y edad.

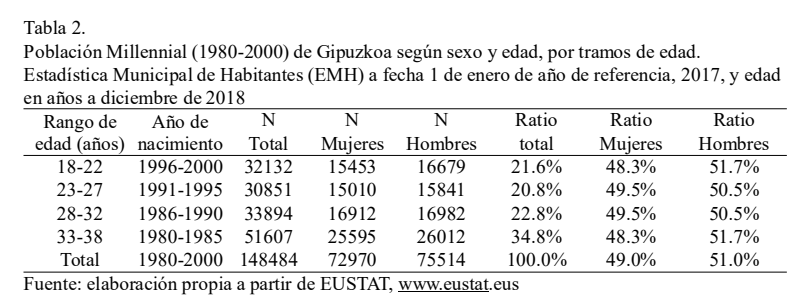

El trabajo de campo se organizó con el objetivo de alcanzar las 1000 respuestas válidas atendiendo, en la medida de lo posible, a las cuotas de género y franjas de edad descritas anteriormente. El cuestionario fue administrado vía on line. La ficha técnica con los detalles de la encuesta puede revisarse en la tabla 3. 


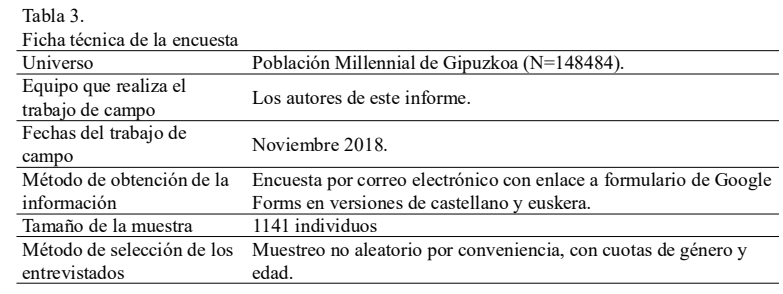

Finalmente, la muestra real obtenida fue la que se describe en la tabla 4. Se observa una sobrerrepresentación de los grupos de edad más joven y de mujeres respecto a la muestra teórica planteada inicialmente en función de las cuotas de género y edad. Asimismo, es preciso indicar que en el cuestionario se recogía la posibilidad de responder con «prefiero no decirlo» al preguntar por el género de la persona encuestada. Si bien es una respuesta muy minoritaria, se ha considerado necesario indicar el nivel de respuesta de esta opción.

En la tabla 4 se describe la muestra según variables como situación laboral, lugar de residencia, nivel de estudios o convivencia.

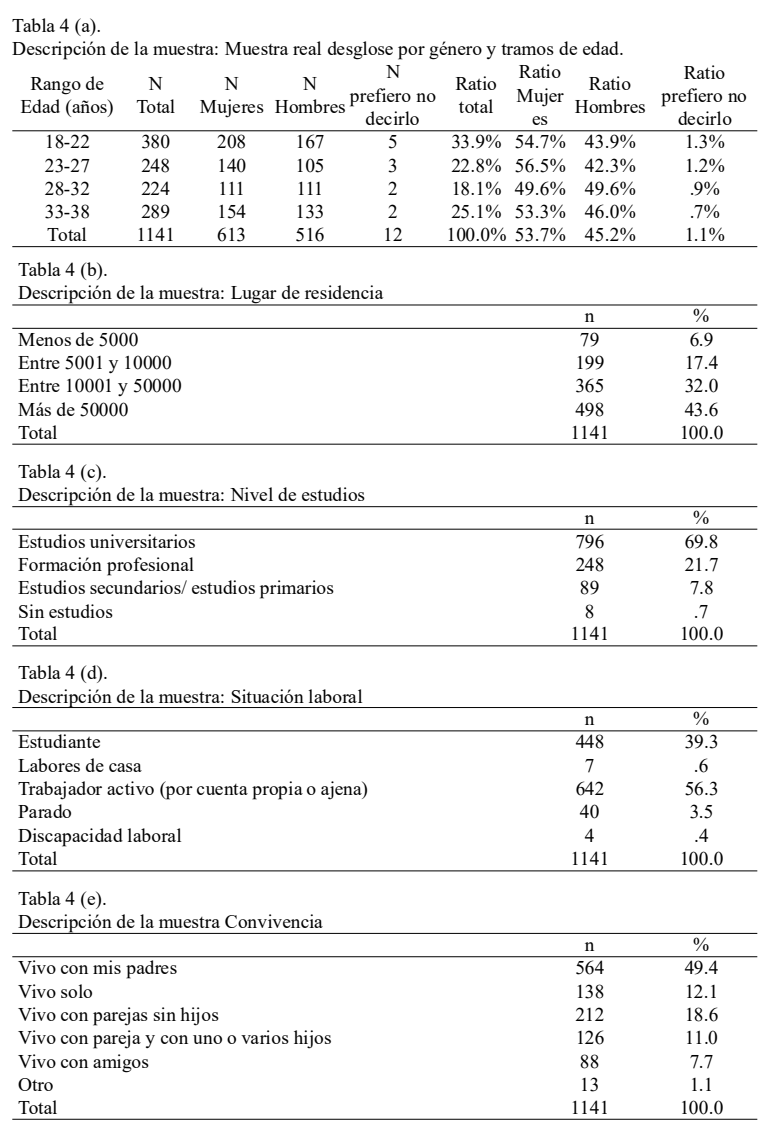

\section{Resultados}

\section{Modelo predictivo del comportamiento deportivo}

En primer lugar, se quiso contrastar que el instrumento de medida utilizado cumplía para la investigación en curso con los requisitos psicométricos de fiabilidad y validez. Para determinar la validez, se realizó un análisis de componentes principales con rotación varimax, donde los dos primeros factores (los únicos con autovalores superiores a 1) explican algo más del $70 \%$ de la varianza (véase tabla 5).

Tabla 5 .

\begin{tabular}{|c|c|c|c|c|c|c|c|c|c|}
\hline \multirow[b]{2}{*}{ Componente } & \multicolumn{3}{|c|}{ Autovalores iniciales } & \multicolumn{3}{|c|}{$\begin{array}{c}\text { Sumas de cargas al } \\
\text { cuadrado de la extracción }\end{array}$} & \multicolumn{3}{|c|}{$\begin{array}{c}\text { Sumas de cargas al } \\
\text { cuadrado de la rotación }\end{array}$} \\
\hline & Total & $\begin{array}{c}\% \text { de } \\
\text { varianza }\end{array}$ & $\begin{array}{c}\% \\
\text { acumulado }\end{array}$ & Total & $\begin{array}{c}\% \text { de } \\
\text { varianza }\end{array}$ & $\begin{array}{c}\% \\
\text { acumulado }\end{array}$ & Total & $\begin{array}{c}\% \mathrm{de} \\
\text { varianza }\end{array}$ & $\begin{array}{c}\% \\
\text { acumulado }\end{array}$ \\
\hline 1 & 7.072 & 58.933 & 58.933 & 7.072 & 58.933 & 58.933 & 5.634 & 46.948 & 46.948 \\
\hline 2 & 1.335 & 11.123 & 70.056 & 1.335 & 11.123 & 70.056 & 2.773 & 23.108 & 70.056 \\
\hline 3 & .543 & 4.528 & 74.584 & & & & & & \\
\hline 4 & .489 & 4.075 & 78.659 & & & & & & \\
\hline 5 & .473 & 3.942 & 82.602 & & & & & & \\
\hline 6 & .425 & 3.539 & 86.141 & & & & & & \\
\hline 7 & .379 & 3.159 & 89.300 & & & & & & \\
\hline 8 & .325 & 2.709 & 92.009 & & & & & & \\
\hline 9 & .293 & 2.445 & 94.454 & & & & & & \\
\hline 10 & .262 & 2.187 & 96.641 & & & & & & \\
\hline 11 & .224 & 1.863 & 98.504 & & & & & & \\
\hline 12 & .180 & 1.496 & 100.000 & & & & & & \\
\hline
\end{tabular}

Las cargas factoriales de todos los ítems alcanzaron su valor máximo (siempre superior a 0.50 ) en uno de estos dos factores. Se distinguieron para el estudio claramente dos factores o dimensiones. Se decidió etiquetar una de las dimensiones como «Amante de la práctica deportiva» y la otra como «Espectador deportivo», distinguiendo ítems que hacen referencia a la propia esencia de la práctica deportiva (los que se cargan en el primer factor) de la actividad placentera de ver programas o eventos deportivos (los que se cargan en el segundo factor).

Tabla 6

Análisis factorial exploratorio. Análisis de componentes principales con rotación Varimax con $\begin{aligned} \text { normalización Kaiser. Cargas factoriales de los ítems en los dos primeros factores } & \\ \text { Amante de la } & \text { Espectador }\end{aligned}$ \begin{tabular}{lc} 
& práctica deportiva \\
\hline La actividad física/deporte ocupa un lugar importante en mi vida. & .848
\end{tabular} Si tuviera una tarde libre emplearía una parte de mi tiempo en $\quad .794$ realizar una actividad fisica o deportiva

Alguna vez me he acostado o levantado antes para poder practicar $\quad .717$

una actividad física/ deporte.

Me gustaria tener siempre un tiempo a la semana para dedicarlo a $\quad .824$

la actividad fisica/deporte.

En relación con mis amigos, dedico más tiempo a la práctica de $\quad .669$

una actividad fisica/deporte.

Si no puedo practicar una actividad física/deporte durante toda $\quad 846$

una semana aumentan las ganas de practicarla.

Cuando veo una actividad fisica/deporte me aumentan las ganas $\quad .691$
de practicarla /o

de practicarla /o

Si dejo de practicar una semana una actividad física/deporte noto

un bajón en mi estado de forma.

a

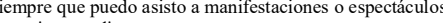

Alguna vez me he acostado o levantado antes para poder ver una

retransmisión deportiva.

Mis programas favoritos son los deportivos.

Seguidamente, se procedió a la comprobación de la fiabilidad de la escala sumativa de ítems asociados a cada dimensión. El coeficiente Alpha de Cronbach para la dimensión llamada «Amante de la práctica deportiva» es de 0.938 y la de «Espectador deportivo» de 0.839 . En ambos casos, los valores son muy superiores al 0.6 recomendado. Además, tal como se observa en la tabla 7 , en ninguno de los casos mejora el Alpha de Cronbach suprimiendo alguno de los ítems. Además, en cada escala, el valor de los coeficientes de correlación ítem-total es siempre superior a 0.68 , muy por encima de los estándares recomendados. La validez y la fiabilidad de la escala de Dosil (2002) queda, pues, contrastada en esta investigación.

Adicionalmente, con el fin de proponer un modelo predictor del comportamiento futuro de la práctica deportiva, se profundizó en el estudio de la influencia sobre la práctica deportiva de algunas de las variables consideradas. Se construyó un primer modelo de regresión logística en el que se tomó como variable dependiente una variable dicotómica practicante/no practicante, construida a partir de la pregunta «Indica con qué frecuencia practicas deporte» con las opciones de respuesta «menos de una vez por semana», «una 
En cuanto a la capacidad predictiva de este primer modelo de regresión logística, cabe señalar que el modelo clasifica correctamente al $82.9 \%$, esto es, a 946 de los 1141 individuos analizados. En concreto, el modelo clasifica correctamente al 90.1\% (647 sobre 718) de los practicantes y al 70.7\% (299 sobre 423) de los no practicantes.

Para poner en perspectiva la importancia de estas proporciones de individuos correctamente clasificados por el modelo, hay que tener en cuenta que en ausencia de todo modelo, y con el conocimiento de la distribución de la variable «práctica deportiva», la proporción de clasificados correctamente podría alcanzar un $62.9 \%$ (718 sobre 1141) empleando el sencillo procedimiento de clasificar a todos en la clase más frecuente (en este caso, practicantes deportivos), mientras que este sencillo

vez por semana», «dos o tres veces por semana», «cuatro o cinco veces por semana», «seis veces por semana»y «todos los días de la semana». Se clasificaron como individuos «no practicantes» los que respondían a la primera y segunda opción, y como individuos «practicantes» aquellos que decían practicar deporte al menos dos o tres veces por semana. El grupo de practicantes quedaba formado por 718 individuos (62.9\%) y el segundo por 423 individuos (37.1\%). Las variables explicativas de este primer modelo se limitaron a las puntuaciones sumadas en los ítems correspondientes a las dos dimensiones de la escala ya validada, «Amante de la práctica deportiva» $\mathrm{y}$ «Espectador deportivo». Los resultados de la estimación de dicho modelo se recogen en la figura 1, en la que para cada uno de los coeficientes del modelo (la constante y los coeficientes propios de cada variable independiente) se muestran su estimación, su error estándar, su valor $\mathrm{t}$ (resultado de la división del valor de la estimación entre el valor del error estándar) y el p-valor asociado al contraste de la hipótesis que afirma que el valor de dicho coeficiente es nulo (y, por tanto, la falta de capacidad predictiva de la variable asociada al coeficiente).

\begin{tabular}{|c|c|c|c|c|}
\hline Coeficientes & Estimate & Std. Error & z value & $\operatorname{Pr}(>|z|)$ \\
\hline (Intercept) & -6.42328 & .41524 & -15.469 & $-<2 \mathrm{e}-16 * * *$ \\
\hline "Amante de la práctica deportiva" & .23590 & .01521 & 15.510 & $<2 \mathrm{e}-16 * * *$ \\
\hline "Espectador deportivo" & -.05062 & .02868 & -1.765 & $0.0776^{*}$ \\
\hline
\end{tabular}

En los resultados se aprecia que, a igualdad de los demás factores, puntuaciones más elevadas en la dimensión «Amante de la práctica deportiva» tiene un efecto positivo y significativo sobre la práctica deportiva $(\mathrm{z}=15.510 ; p=.0000)$. Por el contrario, la dimensión «Espectador deportivo», y siempre a igualdad de los demás factores, presenta un efecto negativo, aunque no significativo; esto es, puntuaciones más elevadas en «Espectador deportivo» están relacionadas, aunque no significativamente en términos estadísticos, con una menor práctica deportiva entre los individuos anali- modelo consigue elevar la proporción de clasificados correctamente desde un $62.9 \%$ hasta un $82.9 \%$, un incremento en absoluto despreciable.

Con el propósito de mejorar la capacidad predictiva del modelo, se estudió uno más complejo que tomó como variables explicativas, además de las puntuaciones sumadas en los ítems correspondientes a las dos dimensiones de la escala ya validada, «Amante de la práctica deportiva» y «Espectador deportivo», el interés por la práctica deportiva(IPD), la autopercepción de salud (SALUD) y la autopercepción como deportista (P). Los resultados de la estimación de este segundo modelo se recogen en la figura 2 . Al igual que en el caso anterior, dicha figura muestra para cada uno de los coeficientes de este segundo modelo (la constante y los coeficientes propios de cada variable independiente) su estimación, su error estándar, su valor $\mathrm{t}$ (resultado de la división del valor de la estimación entre el valor del error estándar) y el pvalor asociado al contraste de la hipótesis que afirma que el valor de dicho coeficiente es nulo (y, por tanto, la falta de capacidad predictiva de la variable asociada al coeficiente).

\begin{tabular}{|c|c|c|c|c|}
\hline Coeficientes & Estimate & Std. Error & $\mathrm{z}$ value & $\operatorname{Pr}(>|z|)$ \\
\hline (Intercept) & -3.30702 & .88087 & -3.754 & $.000174 * * *$ \\
\hline "Amante de la práctica deportiva" & .16288 & .01784 & 9.131 & $<2 \mathrm{e}-16 * * *$ \\
\hline "Espectador deportivo" & -.10109 & .03095 & -3.266 & $.001091 * *$ \\
\hline IPD & .24535 & .06765 & 3.627 & $.000287^{* * *}$ \\
\hline $\mathrm{P}$ & -.63697 & .11934 & -5.337 & $9.43 \mathrm{e}-08 * * *$ \\
\hline SALUD & -.03953 & .06037 & -0.655 & .512567 \\
\hline
\end{tabular}

En los resultados de este segundo modelo se puede apreciar que, a igualdad de los demás factores, puntuaciones más elevadas en la dimensión «Amante de la práctica deportiva» $(\mathrm{z}=9.131 ; p=.000174)$ y en el interés por la práctica deportiva (IPD) $(\mathrm{z}=3.627 ; p=.000287)$ tienen un efecto positivo y significativo sobre la práctica deportiva. Adicionalmente, la autopercepción como deportista $(\mathrm{P})$ presenta un efecto aparentemente negativo $(\mathrm{z}=-5.337 ; p=.0000)$ que debe ser explicado con cierto detalle, ya que debe tenerse en cuenta la 
forma de codificación de dicha variable: cuanto menor es la puntuación que un individuo toma en la variable $\mathrm{P}$, más cercana se encuentra su autopercepción a la de un deportista competitivo; y cuanto mayor es la puntuación que un individuo toma en la variable $P$ más cercana está la autopercepción de dicho individuo a la de un deportista recreativo. En consecuencia, la autopercepción como deportista competitivo muestra un efecto positivo y significativo sobre la práctica deportiva. En lo que se refiere a la autopercepción de salud, ésta no presenta un efecto significativo sobre la práctica deportiva $(\mathrm{z}=-0.655 ; p=.512567)$. Y, por último, al igual que en el primer modelo, puntuaciones más elevadas en «Espectador deportivo» están relacionadas, a igualdad de los demás factores, con una menor práctica deportiva entre los individuos analizados $(\mathrm{z}=-3.266 ; p=.001091)$.

En cuanto a la capacidad predictiva de este segundo modelo de regresión logística elaborado, cabe señalar que el modelo clasifica correctamente al 82.5\%, esto es, 941 de los individuos analizados. En particular, el modelo clasifica correctamente al $88,6 \%$ de los practicantes ( 636 sobre 718 ) y al $72.1 \%$ de los no practicantes ( 305 sobre 423 ).

Este segundo modelo no mejora la capacidad predictiva conjunta $(82.5 \%$ sobre $82.9 \%$ del primer modelo) y muestra una peor capacidad predictiva para los practicantes $(88.6 \%$ sobre $90.1 \%$ del primer modelo). Sin embargo, sí que revela una mejor capacidad predictiva para los no practicantes (72.1\% sobre $70.1 \%$ ). En resumen, este segundo modelo comete menos errores a la hora de clasificar a los no practicantes, pero más con los practicantes (véase la tabla 8).

\begin{tabular}{|c|c|c|c|c|}
\hline & $\begin{array}{c}\text { Clasificados } \\
\text { como } \\
\text { practicantes }\end{array}$ & $\begin{array}{c}\text { Clasificados } \\
\text { como no } \\
\text { practicantes }\end{array}$ & $\begin{array}{c}\text { Total } \\
\text { clasificados }\end{array}$ & $\begin{array}{c}\text { Porcentaje de clasificados } \\
\text { correctamente por el } \\
\text { modelo }\end{array}$ \\
\hline Practicantes & 647 & 71 & 718 & $90.1 \%$ \\
\hline Modelo 1 No practicantes & 124 & 299 & 423 & $70.7 \%$ \\
\hline Total muestra & 771 & 370 & 1141 & $82.9 \%$ \\
\hline Practicantes & 636 & 82 & 718 & $88.6 \%$ \\
\hline Modelo 2 No practicantes & 118 & 305 & 423 & $72.1 \%$ \\
\hline Total muestra & 754 & 387 & 1141 & $82.5 \%$ \\
\hline
\end{tabular}

\section{La actitud hacia la práctica deportiva}

Entrando ya en los resultados propios de la medición, se observa para la muestra en su conjunto una actitud muy favorable hacia la actividad física y práctica deportiva de los Millennials encuestados. Tal y como se indica en la tabla 9, para la dimensión «Amante de la práctica deportiva», formado por nueve ítems valorados en una escala Likert de cinco puntos (con valores de uno a cinco) el valor de la media alcanza 32.04 sobre un valor máximo de 45 y un mínimo de nueve. Merece un lugar especial la respuesta al ítem «la actividad física/deporte ocupa un lugar importante en mi vida», que alcanza una media de 3.74 en una escala de uno a cinco, segunda puntuación más alta de entre todas las referidas a la actitud hacia la actividad física y práctica deportiva. Otros ítems que destacan por haber obtenido una media alta son las referidas a «me gustaría tener siempre un tiempo a la semana para dedicarlo a la actividad física/deporte» (4.11 en una escala de uno a cinco) y «si tuviera una tarde libre emplearía una parte de mi tiempo en realizar una actividad física o deportiva» (3.65 en una escala de uno a cinco). Por el contrario, la media para la dimensión «Espectador deportivo» alcanza el valor de 8.10 sobre un valor máximo de quince y un mínimo de tres, con lo que denota puntuaciones medias más bajas de los ítems que la configuran. Se observa cómo el ítem «mis programas favoritos son los deportivos» alcanza un valor de 2.50 en una escala de uno a cinco y «asistencia a espectáculos deportivos en directo» un valor de 2.68 en una escala de uno a cinco.

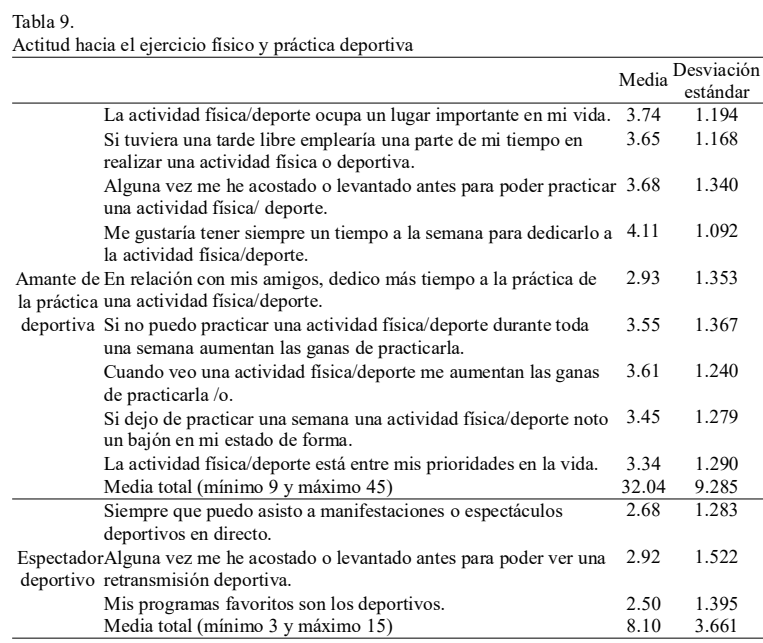

Seguidamente, con objeto de dar respuesta al objetivo de determinar si existen diferencias significativas en la actitud hacia la actividad física y práctica deportiva entre los Millennials que practican deporte y los que no, se analizaron las actitudes de los individuos clasificados en «no practicantes» (423 individuos) y «practicantes» (718 individuos) según el procedimiento explicado previamente.

Para contrastar que aquellos que dicen practicar deporte tiene una mejor actitud hacia la actividad física y práctica deportiva, se llevó a cabo la prueba $T$ para igualdad de medias en muestras independientes con los resultados que se recogen en la tabla 10. Se observa que para todos los ítems las diferencias de actitud hacia la actividad física y práctica deportiva son significativas entre los etiquetados como practicantes (practican deporte al menos dos o tres veces por

\begin{tabular}{|c|c|c|c|c|c|c|}
\hline & & $\begin{array}{l}\text { Practica } \\
\text { deporte }\end{array}$ & Media & Desv. Est. & $\mathrm{t}$ & Sig. (bil.) \\
\hline \multirow{13}{*}{$\begin{array}{l}\text { Amante de } \\
\text { la práctica } \\
\text { deportiva }\end{array}$} & La actividad fisica/deporte ocupa un lugar importante en mi vida. & $\begin{array}{l}\text { Sí } \\
\text { No }\end{array}$ & $\begin{array}{l}4.33 \\
2.73\end{array}$ & $\begin{array}{l}.793 \\
1.079\end{array}$ & 26.578 & .000 \\
\hline & \multirow{2}{*}{$\begin{array}{l}\text { Si tuviera una tarde libre emplearía una parte de mi tiempo en } \\
\text { realizar una actividad física o deportiva. }\end{array}$} & Sí & 4.12 & .867 & \multirow[t]{2}{*}{19.414} & .000 \\
\hline & & $\begin{array}{l}\text { No } \\
\text { Sí }\end{array}$ & $\begin{array}{l}2.85 \\
4.19\end{array}$ & $\begin{array}{l}1.175 \\
1.023\end{array}$ & & \multirow{2}{*}{.000} \\
\hline & $\begin{array}{l}\text { Alguna vez me he acostado o levantado antes para poder practicar } \\
\text { una actividad fisica/ deporte. }\end{array}$ & No & 2.80 & 1.362 & 18.145 & \\
\hline & \multirow{2}{*}{$\begin{array}{l}\text { Me gustaría tener siempre un tiempo a la semana para dedicarlo a la } \\
\text { actividad física/deporte. }\end{array}$} & Si & 4.53 & .719 & \multirow{2}{*}{17.431} & \multirow{2}{*}{.000} \\
\hline & & No & 3.39 & 1.232 & & \\
\hline & \multirow{2}{*}{$\begin{array}{l}\text { En relación con mis amigos. dedico más tiempo a la práctica de una } \\
\text { actividad física/deporte. }\end{array}$} & Sí & 3.46 & 1.207 & 21.080 & .000 \\
\hline & & $\begin{array}{l}\text { No } \\
\text { Sí }\end{array}$ & $\begin{array}{l}2.01 \\
4.19\end{array}$ & $\begin{array}{l}1.009 \\
.960\end{array}$ & \multirow{2}{*}{24.516} & \multirow{2}{*}{.000} \\
\hline & $\begin{array}{l}\text { Si no puedo practicar una actividad física/deporte durante toda una } \\
\text { semana aumentan las ganas de practicarla. }\end{array}$ & No & 2.45 & 1.257 & & \\
\hline & $\begin{array}{l}\text { Cuando veo una actividad física/deporte me aumentan las ganas de } \\
\text { practicarla /o. }\end{array}$ & $\begin{array}{l}\text { Sí } \\
\text { No }\end{array}$ & $\begin{array}{r}4.01 \\
2.92\end{array}$ & $\begin{array}{l}1.017 \\
1.281\end{array}$ & 14.971 & .000 \\
\hline & \multirow{2}{*}{$\begin{array}{l}\text { Si dejo de practicar una semana una actividad física/deporte noto un } \\
\text { bajón en mi estado de forma. }\end{array}$} & Sí & 3.96 & 1.005 & \multirow{2}{*}{19.347} & \multirow{2}{*}{.000} \\
\hline & & No & 2.59 & 1.234 & & \\
\hline & La actividad fisica/deporte está entre mis prioridades en la vida. & $\begin{array}{l}\text { Sí } \\
\text { No }\end{array}$ & $\begin{array}{l}3.91 \\
2.36\end{array}$ & $\begin{array}{l}1.038 \\
1.068\end{array}$ & 23.990 & .000 \\
\hline \multirow{5}{*}{$\begin{array}{l}\text { Espectador } \\
\text { deportivo }\end{array}$} & Siempre que puedo asisto a manifestaciones o espectáculos & Sí & 3.01 & 1.265 & \multirow{2}{*}{12.425} & \multirow{2}{*}{.000} \\
\hline & deportivos en directc & No & 2.12 & 1.107 & & \\
\hline & \multirow{2}{*}{$\begin{array}{l}\text { Alguna vez me he acostado o levantado antes para poder ver una } \\
\text { retransmisión deportiva. }\end{array}$} & Sí & 3.25 & 1.468 & \multirow{2}{*}{10.010} & \multirow{2}{*}{.000} \\
\hline & & No & 2.36 & 1.447 & & \\
\hline & Mis programas favoritos son los deportivos. & Sí & 2.82 & 1.416 & 10.977 & .000 \\
\hline
\end{tabular}


semana) y los no practicantes (practican deporte menos de dos o tres veces por semana), y que la actitud de los primeros es siempre más favorable que la de los segundos. De esta forma, queda contrastada la idea planteada de que tienen mejor actitud hacia la actividad física y práctica deportiva aquellos individuos Millennials que actualmente dicen ser practicantes que los que no (véase la tabla 10).

Finalmente, para responder al siguiente objetivo que trata de determinar si existen diferencias en la actitud hacia la actividad física y práctica deportiva entre los Millennials hombres (516 individuos, esto es, $45 \%$ de la muestra) y mujeres (613 individuos, esto es, 55\% de la muestra), se procedió de la misma manera. Se llevó a cabo la prueba T para igualdad de medias en muestras independientes con los resultados que se recogen en la tabla 11. En este caso, se observa que en ninguno de los ítems de la dimensión etiquetada como «Amante de la práctica deportiva» hay diferencias entre hombres y mujeres y solo en un ítem de la dimensión «Espectador deportivo» hay diferencias significativas al 5\%. De esta forma, se puede afirmar que no hay evidencias suficientes de que haya diferencias en la actitud hacia la actividad física y práctica deportiva entre hombres y mujeres.

De todas formas, y aunque las diferencias no sean significativas, sí cabe anotar que las puntuaciones medias de las mujeres son más altas que las de los hombres en todos los ítems, excepto para «si tuviera una tarde libre emplearía una parte de mi tiempo en realizar una actividad física o deportiva». En resumen, aun no siendo las diferencias significativas, sí se intuye cierta mejor actitud hacia la práctica deportiva entre las mujeres que entre los hombres (véase la tabla 11).

\begin{tabular}{|c|c|c|c|c|c|c|}
\hline & & Sexo & Media & Desv. Est. & $\mathrm{t}$ & Sig. (bilateral) \\
\hline \multirow{16}{*}{$\begin{array}{l}\text { Amante de } \\
\text { la práctica } \\
\text { deportiva }\end{array}$} & \multirow[t]{2}{*}{ La actividad fisica/deporte ocupa un lugar importante en mi vida. } & Mujer & 3.75 & 1.184 & \multirow{2}{*}{.515} & \multirow{2}{*}{.607} \\
\hline & & Hombre & 3.72 & & & \\
\hline & \multirow{2}{*}{$\begin{array}{l}\text { Si tuviera una tarde libre emplearía una parte de mi tiempo en } \\
\text { realizar una actividad fisica o deportiva. }\end{array}$} & Mujer & 3.64 & 1.183 & \multirow{2}{*}{-.178} & \multirow{2}{*}{.859} \\
\hline & & Hombre & 3.66 & 1.139 & & \\
\hline & \multirow{2}{*}{$\begin{array}{l}\text { Alguna vez me he acostado o levantado antes para poder practicar } \\
\text { una actividad fisica/ deporte. }\end{array}$} & Mujer & 3.70 & 1.330 & \multirow{2}{*}{.660} & \multirow{2}{*}{.509} \\
\hline & & Hombre & 3.65 & 1.348 & & \\
\hline & \multirow{2}{*}{$\begin{array}{l}\text { Me gustaría tener siempre un tiempo a la semana para dedicarlo a la } \\
\text { actividad fisica/deporte. }\end{array}$} & Mujer & 4.15 & 1.071 & \multirow{2}{*}{1.719} & \multirow{2}{*}{.086} \\
\hline & & Hombre & 4.04 & 1.112 & & \\
\hline & \multirow{2}{*}{$\begin{array}{l}\text { En relación con mis amigos. dedico más tiempo a la práctica de una } \\
\text { actividad fisica/deporte. }\end{array}$} & Mujer & 2.99 & 1.356 & \multirow{2}{*}{1.884} & \multirow{2}{*}{.060} \\
\hline & & Hombre & 2.84 & 1.335 & & \\
\hline & $\begin{array}{l}\text { Si no puedo practicar una actividad fisica/deporte durante toda una } \\
\text { semana aumentan las ganas de practicarla. }\end{array}$ & Mujer & $\begin{array}{l}3.56 \\
3.52\end{array}$ & $\begin{array}{l}1.374 \\
1.354\end{array}$ & .506 & .613 \\
\hline & \multirow{2}{*}{$\begin{array}{l}\text { Cuando veo una actividad física/deporte me aumentan las ganas de } \\
\text { practicarla /o. }\end{array}$} & Mujer & 3.64 & 1.260 & \multirow{2}{*}{.923} & \multirow[b]{2}{*}{.356} \\
\hline & & Hombre & 3.57 & 1.214 & & \\
\hline & \multirow{2}{*}{$\begin{array}{l}\text { Si dejo de practicar una semana una actividad física/deporte noto un } \\
\text { bajón en mi estado de forma. }\end{array}$} & $\begin{array}{l}\text { Mujer } \\
\text { Hombre }\end{array}$ & 3.48 & $\begin{array}{l}1.270 \\
1284\end{array}$ & \multirow[t]{2}{*}{.842} & \multirow{2}{*}{.400} \\
\hline & & Hombre & 3.42 & 1.284 & & \\
\hline & La actividad física/deporte está entre mis prioridades en la vida. & Mujer & 3.37 & 1.281 & .897 & .370 \\
\hline & \multirow{2}{*}{$\begin{array}{l}\text { Siempre que puedo asisto a manifestaciones o espectáculos } \\
\text { deportivos en directo. }\end{array}$} & & 2.72 & & \multirow[b]{2}{*}{1.395} & \multirow[b]{2}{*}{.163} \\
\hline & & Hombre & 2.62 & 1.263 & & \\
\hline \multirow{3}{*}{\multicolumn{2}{|c|}{$\begin{array}{l}\text { Espectador Alguna vez me he acostado o levantado antes para poder ver una } \\
\text { deportivo retransmisión deportiva. } \\
\text { Mis programas favoritos son los deportivos. }\end{array}$}} & Mujer & 3.05 & 1.536 & \multirow{2}{*}{3.314} & \multirow{2}{*}{.001} \\
\hline & & Hombre & 2.75 & 1.487 & & \\
\hline & & Mujer & 2.57 & 1.435 & 1.777 & .076 \\
\hline & & Hombre & 2.42 & 1.344 & & \\
\hline
\end{tabular}

\section{Discusión}

Conocedores de los beneficios que tiene la realización de ejercicio físico en la salud y los cambios que se producen en los hábitos de la práctica deportiva en individuos que abandonan la juventud para entrar en la etapa adulta, esta investigación se ha centrado en el colectivo de los Millennials, conformado por la cohorte demográfica nacida entre 1980 y 2000. Este segmento supone cerca de una cuarta parte de la población con unos rasgos muy determinados por la digitalización que lo convierten en colectivo de especial interés y relevancia. El objetivo ha sido doble, uno metodológico y otro más de caracterización del grupo.

A nivel metodológico, se han contrastado los requisitos psicométricos de la escala de la actitud hacia la actividad física y deportiva de Dosil (2002), avanzando respecto a la literatura existente en la bi-dimensionalidad del constructo. La investigación apunta hacia la existencia de un factor formado por variables con connotación activa frente a otro de variables más pasivas, y que se han etiquetado como «Amante de la práctica deportiva» y «Espectador deportivo». En esta misma línea, Cruz Santos, González Rivera \& Rosario Rodríguez (2019) determinaron hasta tres dimensiones en una muestra de estudiantes puertorriqueños, si bien es cierto que la escala utilizada difiere en cierta medida de la de Dosil. Se ha contrastado la fiabilidad y validez en un grupo de individuos que difiere de otras investigaciones previas realizadas en otros grupos de edad y ámbitos geográficos distintos. Esta bi-dimensionalidad podría ser contrastada en investigaciones que se animen a utilizar fielmente la escala de Dosil (2002) para confirmar esta cualidad manifestada en este estudio.

A esta aportación metodológica hay que sumarle otra de carácter predictivo. La técnica de regresión logística introduce en el estudio de la actitud hacia la actividad física y el deporte una novedad, en la medida que demuestra que la escala de dicho constructo es un buen predictor de la variable dicotómica practicante/no practicante de un individuo, sola o combinada con variables como la práctica deportiva, la autopercepción de la salud o la autopercepción como deportista. En definitiva, con la aplicación de la técnica de regresión logística se ha conseguido un modelo con una elevada capacidad predictiva para clasificar individuos practicantes y no practicantes a partir de la actitud hacia el ejercicio físico y práctica deportiva.

A nivel de medición de la actitud, los Millennials participantes en esta investigación muestran una actitud hacia la actividad física y el deporte muy favorable, de manera que, según estos resultados, cabría sugerir que este grupo, además de caracterizarse por ser «nativos digitales», «multipantalla y multidispositivo», «nomófobos y appdictos», extremadamente sociales y «super conectados», críticos, exigentes, volátiles, orientados hacia hábitos saludables, alegres, tranquilos y optimistas (BBVA Innovation Center, 2015, Fundación Mapfre, 2019), son «Amantes de la práctica deportiva»y «Espectadores deportivos». Los favorables resultados actitudinales son coincidentes con estudios precedentes (véase Tereza Araujo y Dosil, 2016 y los trabajos citados por ellos). A todos esos hallazgos quizá habría que añadir la «novedad» de que esta generación, con particularidades en términos de digitalización, sigue mostrando la misma actitud positiva frente al ejercicio físico y la práctica deportiva. 
Además, la investigación ha podido determinar que los individuos que dicen practicar deporte muestran una actitud hacia la actividad física y práctica del deporte mejor que aquellos que dicen no practicarlo de manera significativa en términos estadísticos. Este resultado es también coherente con las investigaciones realizadas previamente y citadas por Tereza Araujo y Dosil (2016). Sin embargo, y aunque no se han observado diferencias significativas en términos estadísticos, los datos sí apuntan hacia una mejor actitud entre las mujeres Millennials encuestadas que entre los hombres. Este dato contrasta con resultados de otras investigaciones que vienen a concluir que los hombres muestran una mejor actitud que las mujeres (entre los más recientes, véanse, por ejemplo, Fraquela-Vale, Varela-Garrote, \& Varela-Crespo, 2020 o Herazo-Beltran, Nuñez-Bravo, Sánchez-Guette, VásquezOsorio, Lozano-Ariza, Torres-Herrara, \& Valdelamar-Villegas, 2020) Este resultado podría sugerir un cambio en la generación Millennial en comparación con otros colectivos estudiados hasta ahora, donde la actitud siempre ha sido mejor entre los hombres que entre las mujeres.

\section{Conclusión}

El estudio pretende avanzar en la literatura ya existente, contrastando la bi-dimensionalidad de una escala previamente validada y centrando su mirada en el colectivo de los Millennials. Su valor no radica tanto en la comparación con otras generaciones sino en la necesidad de predecir los comportamientos del futuro, siendo esencial desarrollar actitudes favorables que promuevan hábitos saludables beneficiosos para el individuo y la comunidad en su conjunto. El modelo predictivo propuesto permite disponer de información que caracteriza a este grupo y supone un recurso sumamente relevante para que las estructuras económicas, sociales, culturales, políticas y medios de comunicación puedan incidir en estrategias, políticas, programas y acciones en pro de una sociedad cada vez más orientada a la práctica deportiva. En esta línea, sería interesante profundizar en las motivaciones y frenos para la práctica de ejercicio físico de los Millennials hombres y mujeres.

En cualquier caso, debe hacerse notar la principal debilidad de este estudio, la muestra, que, aunque amplia y variada, no ha sido aleatoria, sino de conveniencia, aspecto que impide la generalización de los resultados. No obstante, la amplitud de la muestra y la forma de seleccionar las unidades muestrales (muestreo por cuotas de sexo y edad) invitan a pensar que debe tener las características de una muestra pseudoaleatoria. Adicionalmente, el tamaño de la muestra utilizada también ha sido superior al de la mayoría de las investigaciones precedentes.

\section{Conflicto de intereses}

Las autorías no han comunicado ningún conflicto de intereses.

\section{Referencias}

Araújo, D., Calmeiro, L., \& Palmeira, A. (2005). Intenções para a prática de actividades físicas. Cuadernos De Psi- cología Del Deporte, 5, 1-2. Recuperado de https:// revistas.um.es/cpd/article/view/93551

BBVA Innovation Center (2015). Así es la primera generación completamente digital. Generación 'Millennial'. Serie Innovation Trends. Junio. Recuperado de: www.centrodeinnovacionbbva.com

Biddle, S., \& Mutrie, N. (2008). Psychology of physical activity: determinants, well-being and interventions (Segunda Edición). Londres: Springer-Verlag.

Caetano, L. M., \& Vasconcelos Raposo, J. (2005). Atitudes dos idosos face à actividade física. Cuadernos De Psicología Del Deporte, 5, 1-2. Recuperado de https:// revistas.um.es/cpd/article/view/93461

Cid, L., \& Alves, J. (2008). Attitude toward physical activity. Differences between gender. En J. Cabri, F. Alves, D. Araújo, J. Barreiros, J. Diniz, \& A. Veloso (Eds.). Book of abstracts 13th Annual Congresso of the European College of Sport Science. Lisboa: ECSS-FMH, 415.

Cid, L., Chicau, C., Silva, C., \& Moutão, J. (2009). Influência do género, idade, condição de praticante e o tipo de actividade praticada. En J. Dosil, J. Diaz y I. Diaz (Eds.). Book of abstracts II Congresso da Sociedade Iberoamericana de Psicologia do Desporto [CD-ROM]. Torrelavega, España.

Cruz Santos, A. A., González Rivera, J. A., \& Rosario Rodríguez, A. (2019). Actitud sobre el ejercicio físico y los deportes: Un estudio psicométrico en estudiantes universitarios. Revista Evaluar, 19(2). https://doi.org/ 10.35670/1667-4545.v19.n2.25083

Dosil Díaz, J. (2002). Escala de actitudes hacia la actividad física y el deporte (E.A.F.D.). Cuadernos De Psicología Del Deporte, 2(2). Recuperado de https://revistas.um.es/ cpd/article/view/112241

EUSTAT. www.eustat.eus

Fermino, R.C., Pezzini, M. R., \& Reis, R. S. (2010). Motivos para prática de atividade física e imagem corporal em frequentadores de academia. Revista Brasileira de Medicina do Esporte, 16(1), 18-23. https://dx.doi.org/ 10.1590/S1517-86922010000100003

Fondo Mundial para la Investigación del Cáncer/Instituto Estadounidense de Investigación sobre el Cáncer. (2007). Alimentos, nutrición, actividad física, y la prevención del cáncer: una perspectiva mundial. Washington, D.C.: AICR, 2007. Recuperado de https://www.wcrf.org/sites/ default/files/spanish.pdf

Fraguela-Vale, R., Varela-Garrote, L. \& Varela-Crespo, L. (2020). Perfiles de ocio deportivo en jóvenes españoles (15-20 años): un análisis de género. Retos, 37, 419-426.

Fundación Mapfre. (2019). Millennials y Salud. Recuperado de https://www.fundacionmapfre.org/documentacion/ p u b l i c o / e s / c a t a 1 o g o _ i m a g e n e s / imagen.do?path $=1103996$ \&posicion $=2$ \&registrardownload $=1$

Gómez, M.M., Sánchez, O.D., Labisa, P.A. (2020). Actividad física en tiempo libre en estudiantes universitarios colombianos. Retos, 37(1), 181-189.

Haase, A. M., \& Kinnafick, F. E. (2007). What factors drive regular exercise behavior?: Exploring the concept and maintenance of habitual exercise. Journal of Sport Exercise Psychology, 29(Suppl.), S165. 0895-2779. Recuperado de http://nectar.northampton.ac.uk/6214/ 
Hagger, M., Chatzisarantis, N., \& Biddle, S. (2001). Studying the influence of attitudes, intentions and past behaviour on leisure time exercise participation - Predictive validity using meta analysis. En A. Papaioannou, M. Goudas, \& Y. Theodorakis (Eds.). Proceedings of $10^{\text {th }}$ World Congress of Sport Psychology: In the Down of the New Millennium (98-100). Skiathos-Greece: Christodoulides Publications.

Herazo-Beltran, Y., Nuñez-Bravo, N., Sánchez-Guette, L., Vásquez-Osorio, F., Lozano-Ariza, A., Torres-Herrera, E., Valdelamar-Villegas, A. (2020). Estilos de vida relacionados con la salud en estudiantes universitarios. Retos, 38, 547-551.

Lima, L. (2000). Atitudes: Estrutura e mudança. En: J. Vala, \& M. Monteiro(Eds.). Psicologia Social (187-225). Lisboa: Fundação Calouste Gulbenkian.

Martin, J. J., Oliver, K., \& McCaughtry, N. (2007). The theory of planned behavior: Predicting physical activity in Mexican American children. Journal of Sport and Exercise Psychology, 29(2), 225-238. Recuperado de: http://digitalcommons.wayne.edu/coe_khs/20

Meneses, M., \& Ruiz, F. (2017). Estudio longitudinal de los comportamientos y el nivel de actividad físico-deportiva en el tiempo libre en estudiantes de Costa Rica, México y España. Retos, 31(1), 219-226.

Ministerios de Educación, Cultura y Deporte. Encuesta de Hábitos Deportivos en España 2015. Madrid, 2015. Recuperado de: https://www.culturaydeporte.gob.es/dam/ jcr:aa63cca9-31 a5-47ce-8ac2-105215f64d9f/ Encuesta de Habitos Deportivos 2015 Sintesis de Resultados.pdf

Nielsen Sports (2018). Nativos Digitales, Millenials y Deporte. Abril. Recuperado de: https://nielsensports.com/ es/millenials-nativos-digitales-y-deporte-2018/

Ornelas-Melim, F. M., \& Oliveira-Pereira, B. (2013). Prática desportiva, um meio de prevenção do bullying na escola? Movimento, 19(2), 55-77. Recuperado de: https:// www.redalyc.org/articulo.oa?id=1153/115326317016

Pavón, A., \& Moreno, J. A. (2008). Actitud de los universitarios ante la práctica físicodeportiva: diferencias por género. Revista de Psicología del Deporte, 17(1), 7-23. Recuperado de: https://www.redalyc.org/ articulo.oa?id=235119246001

R Core Team (2019). R: A language and environment for statistical computing. R Foundation for Statistical Computing, Vienna, Austria. URL https://www.Rproject.org/.

Ruiz, F. J., García, M. E. M., \& Díaz, A. S. (2007). Análisis de las motivaciones de práctica de actividad física y de abandono deportivo en la Ciudad de La Habana (Cuba). Anales de Psicología, 23(1), 152-166. Recuperado de: https:/ /www.redalyc.org/articulo.oa?id=16723119

Salles-Costa, R., Heilborn, M. L., Werneck, G. L., Faerstein, E., \& Lopes,C. S. (2003). Gênero e prática de atividade física de lazer. Cadernos de Saúde Pública, 19(2), S325S333. Recuperado de: http://www.scielo.br/ scielo.php?script=sci_arttext $\&$ pid $=$ S 0102 311X2003000800014

Sicilia, A. C., Águila, C. S., Muyor, J. M. R., Orta, A. C., \& Moreno, J. A. M. (2009). Perfiles motivacionales de los usuarios en centros deportivos municipales. Anales de Psicologia, 25(1), 160-168. Recuperado de: https:// www.um.es/analesps/v25/v25 1/18-25_1.pdf

TerezaAraújo, A., \& Dosil, J. (2015). The influence of attitudes toward physical activity and sports. Motriz, Rio Claro, 21(4), 344-351, Oct/Dec. 2015. Recuperado de: http:// www.scielo.br/pdf/motriz/v21n4/1980-6574-motriz-21-0400344.pdf

Tereza Araújo, A., \& Dosil, J. (2016). Relaciones entre actitudes y práctica de actividad física y deporte en hombres y mujeres. Cuadernos de Psicología del Deporte, 16(3), 67-72. Recuperado de: http://scielo.isciii.es/pdf/cpd/ v16n3/psicologia_deporte6.pdf

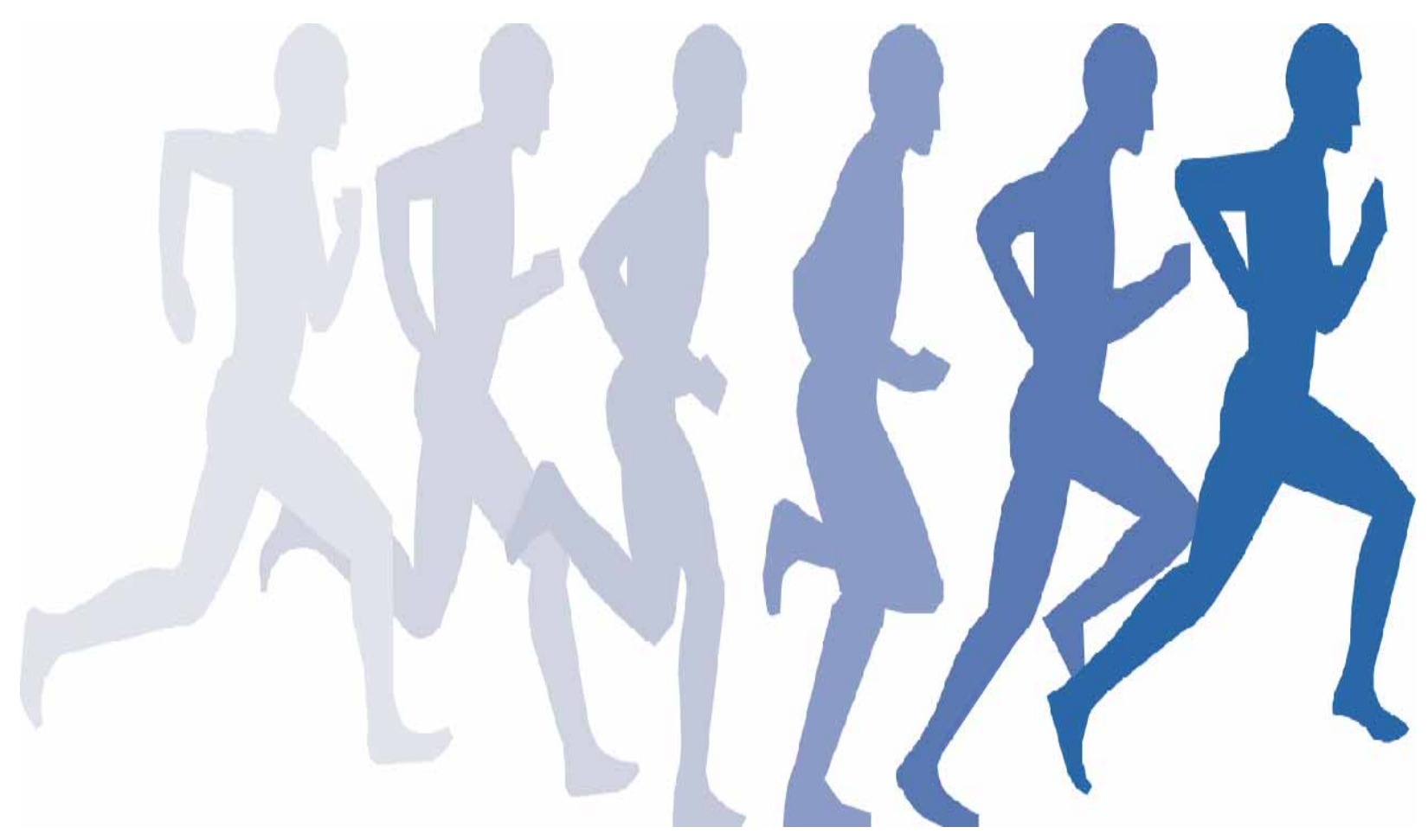

